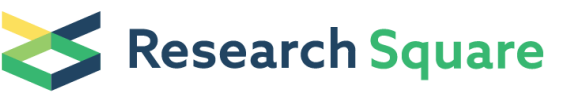 \\ Preprints are preliminary reports that have not undergone peer review. \\ They should not be considered conclusive, used to inform clinical practice, or referenced by the media as validated information.
}

\section{Characterization of Long Non-coding RNA Profile in RD and SH-SY5Y Cells Infected by CV-B5 using RNA Sequencing}

\section{Wei Chen ( $\sim 55685836 @ q q . c o m$ )}

Kunming University of Science and Technology https://orcid.org/0000-0002-6728-4277

\section{Peiying Teng}

Kunming University of Science and Technology

Heng Yang

Kunming University

Jing Li

Kunming University of Science and Technology

\section{Fan Yang}

Kunming University of Science and Technology

\section{Research Article}

Keywords: Coxsackievirus B5 (CV-B5), long noncoding RNAs (IncRNAs), human rhabdomyosarcoma (RD) cell, SH-SY5Y cell, neurological damages

Posted Date: July 27th, 2021

DOI: https://doi.org/10.21203/rs.3.rs-735324/v1

License: (c) (i) This work is licensed under a Creative Commons Attribution 4.0 International License. Read Full License 


\section{Abstract}

Hand, foot, and mouth disease caused by Coxsackievirus B5 (CV-B5) poses considerable threats to the health of infants especially in neurological damage. And the long noncoding RNAs (IncRNAs) act pivotal factors in regulating and participating in virus-host interactions. However, the role of IncRNAs in CV-B5host interactions has not yet been elucidated. In this study, we used the RNA sequencing to determine the expression profiles of IncRNAs in CV-B5 infected human rhabdomyosarcoma (RD) and SH-SY5Y cells. Our results identified that in the differentially expressed IncRNAs a total of 508 up-regulated and 760 down-regulated IncRNAs in RD cell, with $46.2 \%$ were lincRNAs, $28.6 \%$ were anti-sense IncRNAs, $24.1 \%$ were sense overlapping IncRNAs, and 1.0\% were sense intronic IncRNAs. Moreover, 792 IncRNAs were significantly increased and 811 IncRNAs were greatly decreased in SH-SY5Y cell including 48.6\% were lincRNAs, $34.7 \%$ were anti-sense IncRNAs, $16.0 \%$ were sense overlapping IncRNAs, and $0.8 \%$ were sense intronic IncRNAs. Gene Ontology (GO) and Kyoto Encyclopedia of Genes and Genomes (KEGG) pathway showed that the differentially expressed IncRNAs participated in the occurrence of disease in RD cell and associated with signaling pathway in SH-SY5Y cell after CVB5 infection respectively. In addition, similar results were obtained when seven IncRNAs were selected for validation using RT-qPCR assays. Moreover, we conducted the candidate InCRNA-IL12A secondary structures and found that it inhibits viral replication through Wnt signaling pathway. Our results reveal that IncRNAs can become a possible novel molecular target for the prevention and treatment of CV-B5 infection, and provided information for distinguishing neurogenic CV-B5 disease.

\section{Introduction}

Hand, foot, and mouth disease (HFMD) is a common contagious disease among young children especially under the age of five years. HFMD usually occurs in the spring to fall and follows a mild and self-limiting course, such as fever and papulovesicular rash on the hands and feet, mouth [1]. Severe complications such as the central nervous system and significant mortality have also been observed in a small proportion of children suffering from $\operatorname{HFMD}[2,3]$. The main pathogens of HFMD are enterovirus and coxsackievirus. Studies conducted in the past ten years had reported that enterovirus A71 (EV-A71) and coxsackievirus A16 (CV-A16) were the two major pathogens worldwide [4, 5]. However, in recent years the incidence of HFMD caused by coxsackievirus B5 (CV-B5) has increased. CV-B5 had been widely associated with both sporadic cases and outbreaks of HFMD worldwide, particularly in China, South East Asia and Europe [6-8]. CV-B5 infection not only caused severe HFMD, but also the neurological damages, including aseptic meningitis, viral encephalitis, and acute flaccid paralysis [9-11]. CV-B5 belongs to the Enterovirus genus of the Picornaviridae family and is a positive-sense single-stranded RNA with approximately $7500 \mathrm{bp}$. However, there is no effective vaccine for the diseases caused by CV-B5 nor the mechanism of CV-B5 association with the nervous system has yet been elucidated.

Long non-coding RNAs (IncRNAs) are non-coding RNAs (ncRNAs) that are greater than 200 nucleotides in length and do not have protein coding ability. They are classified into several categories including sense, antisense, bidirectional, intronic and intergenic IncRNA based on the location of IncRNAs and target gene 
on chromosome $[12,13]$. LncRNAs are versatile molecules that can interact physically and functionally with DNA, other RNAs, and proteins, either through nucleotide base pairing or via formation of structural domains generated by RNA folding [14]. Previous studies have reported that IncRNAs regulate various aspects of an organism's life including the cell cycle and differentiation, epigenetic regulation of genome expression, transcription and post-transcriptional mRNA regulation, and protein translation $[15,16]$.

High throughput RNA sequencing has proved that IncRNAs also regulated and participated in virus-host interactions. Some studies have also reported that IncRNAs are involved in innate immune responses by regulating innate immune cells and transcription of gene expression programs $[17,18]$. In addition, they regulated viral life cycle and modulated host transcription, thereby promoting viral replication. Indeed, some of the earliest characterized IncRNAs were those transcribed from viral genomes $[19,20]$. LncRNAs can also affect downstream signaling pathways and change the interaction between host and virus [21, 22]. Therefore, many researches have showed that IncRNAs were associated with virus replication and infection. However, the function of IncRNAs in CV-B5 infection is still unknown. Therefore, understanding the relationship between the CV-B5 and IncRNAs may reveal the potential targets for antiviral drugs and provide insights for disease affecting the central nervous system.

In this study, RNA sequencing was used to determine the expression of IncRNAs with or without CV-B5 infection in human rhabdomyosarcoma(RD)cell and the human neuroblastoma-derived neuronal cell lineSH-SY5Y cell. And Gene Ontology (GO) and Kyoto Encyclopedia of Genes and Genomes (KEGG) pathways were adopted to define the potential role of differentially expressed IncRNA in CV-B5-host interactions. We also further conducted the candidate IncRNA-IL12A to elucidate its effect on CV-B5 proliferation. Our results will identify the novel molecular pathways for the treatment of severe HFMD.

\section{Materials And Methods}

\section{Cells and CV-B5 virus infection}

RD and SH-SY5Y cells were cultured in Dulbecco's Modified Eagle Medium (DMEM) (SH30243.01, HyClone) supplemented with $10 \%$ fetal bovine serum (FBS) (FBSSA500-S, AusGeneX) or $2 \%$ FBS (maintenance medium) at $37^{\circ} \mathrm{C}$ in a humidified incubator with $5 \% \mathrm{CO}_{2}$. The $\mathrm{CV}-\mathrm{B} 5$ virus was stored at $-80^{\circ} \mathrm{C}$ in our laboratory. When RD and SH-SY5Y cells were cultured approximately to $80 \%$ confluency and then were infected with CV-B5 virus at multiplicity of infection (MOI) of 1 . The cytopathic effect (CPE) in $\mathrm{RD}$ and SH-SY5Y cells were evaluated at 24-hour post-infection (hpi) by imaging under an electron microscope. At the same time, virus infection was verified using the CV-B5 specific antibody (preserved in our laboratory).

\section{RNA isolation}

Total RNA was extracted from virus uninfected and infected RD and SH-SY5Y cells using RNAiso Plus (No.9109, TaKaRa) according to the manufacturer's instructions. RNA quality was evaluated on $1 \%$ 
agarose gels, followed by determination of the concentration and purity by measuring the absorption at $260 \mathrm{~nm}$ using a Nano Photometer spectrophotometer. Total RNA obtained from the samples was used for the subsequent steps.

\section{RNA library construction and sequencing}

Ribosomal RNA was first eliminated, and first strand cDNA and second strand cDNA were synthesized using random hexamer primers [23]. It is worth noting that the dTTP in dNTPs is replaced by dUTP in the synthesis of the second chain, followed by end repair, poly-A 3 ' ends, ligation of adapters, and screening of DNA fragments in order to construct the RNA-seq library [24]. Library quality was assessed using Qubit2.0, Agilent 2100, and RT-PCR [25], while next-generation high throughput sequencing of samples were performed on the Illumina PE $150[26,27]$. The RNA library was sequenced by Novogene Co., LTD (Beijing, China).

\section{Assessment and classification of new IncRNAs}

High quality clean data was assembled, and then the novel transcripts were filtered in order to remain with transcripts whose length was greater than $200 \mathrm{bp}$ and had at least two exons. CPC2, CNCl, and PFAM tools were then used to predict the coding ability of the transcripts. Previous studies have reported that an intersection of the tools indicates novel IncRNA [28, 29]. Then we calculated expression levels of the transcripts using fragments per kilobase of transcript per million fragments mapped (FPKM) values [30]. Differentially-expressed genes were defined as $p<0.05$ and/or fold change $>2$ times based on their FPKM values between the groups, which were calculated using Cuffdiff soft-ware [31]. Differentiallyexpressed IncRNAs are divided into four classes: antisense, intergenic, sense overlapping, and sense intronic IncRNAs according to their position relative to protein-coding genes [32].

\section{GO annotations and KEGG enrichment}

Gene ontology (GO) is a comprehensive database for describing molecular functions, biological processes, and cell composition because it provides the functional annotation and classification of molecular functions [33]. On the other hand, the Kyoto Encyclopedia of Genes and Genomes (KEGG) is a resource database that integrates information on genome, chemistry, and system functions [34]. We performed GO and KEGG enrichment analysis on target genes in order to determine the co-expression of differentially-expressed genes.

\section{RT-qPCR confirmation}

RNA was verified using a One Step TB Green PrimeScript RT-PCR kit (RR086A, Takara). Reactions were performed using an Applied Biosystems 7500 Real Time PCR System (Applied Biosystems), with the 
following cycle conditions: $42^{\circ} \mathrm{C}$ for $5 \mathrm{~min}, 95^{\circ} \mathrm{C}$ for $10 \mathrm{~s}, 40$ cycles at $95^{\circ} \mathrm{C}$ for $5 \mathrm{~s}$, and $60^{\circ} \mathrm{C}$ for $34 \mathrm{~s}$. GAPDH from Homo sapiens was chosen as the endogenous reference gene. All the primers used in the study are listed in Table 1.

Table 1

Primes used for Real-time RT-PCR

\begin{tabular}{|lll|}
\hline Gene Name & Forward Primer[5'-3'0 & Reverse Primer[5'-3'0 \\
\hline SMIM18 & CGCAACTGTATAAACAACTGGACT & AACGTTCAGCATCTTCTGAGTC \\
\hline IL12A & CGAGCGAGCCAAAGACCTG & GGCAAGATAAGCCCAACTGC \\
\hline IL17B & CACAGGTCAAAGAAGCCTCC & GGGCAGGGCAAATGTCCAAC \\
\hline LNC428 & AAGTACACAGCGTCATTCGT & AGGTCCAAATATCCACTTGCAG \\
\hline JAM3 & AGACCTCAGCTTCCTCTGTCAC & CGGCGCGGCTTGTAGT \\
\hline RELA & AAGAGGACATTGAGTTCTGTCCC & TGCCCAGAAGGAAACACCAT \\
\hline GAB1 & CAATGACCCTGAGAGCTGGC & TCACTTGGAAATGCTCGTGGAA \\
\hline GAPDH & GTATGACAACGAATTTGGCTACAG & AGCACAGGGTACTTTATTGATGG \\
\hline
\end{tabular}

\section{Analysis of the candidate IncRNA-IL12A}

A significantly up-regulated IncRNA-IL12A was only obtained in CV-B5 infected SH-SY5Y cell. Using RNAfold predicted the structure of IncRNA-IL12A, and verified its influence on virus replication.

\section{Results}

\section{Validation of the CV-B5 infection models}

$\mathrm{RD}$ and $\mathrm{SH}-\mathrm{SY} 5 \mathrm{Y}$ cells infected with $1 \mathrm{MOI} \mathrm{CV}-\mathrm{B} 5$ at $24 \mathrm{~h}$ can cause $\mathrm{CPE}$, including rounding up, aggregation, and even death when compared with control cell (Fig. 1A). Western Bolt analysis also detected the viral specific protein VP1 in both RD and SH-SY5Y cells (Fig. 1B). Three independent experiments were carried out. These results showed that RD and SH-SH5Y cells were successfully permissive to CV-B5 infection.

\section{Analysis and classification of differentially-expression of IncRNAs}


To analyze the level of transcripts, RD and SH-SY5Y cells infected with CV-B5 were subjected to Human Genome Array. We obtained 6500 and 5375 novel IncRNAs in RD and SH-SY5Y cells respectively, based on $\mathrm{CPC} 2, \mathrm{CNCl}$ and PFAM analysis (Fig. 2A and 2B). With regard to the differentially-expressed genes, our transcript contained a total of 1268 IncRNAs in RD cell (Supplementary table 1) and 1603 IncRNAs in SHSY5Y cell (Supplementary table 2). In total, 1264 IncRNAs with transcripts length $\geq 200$ bp and multi-exon in nature were acquired from the primary IncRNAs in RD cell, of which, $46.2 \%$ were lincRNAs, $28.6 \%$ were anti-sense IncRNAs, 24.1\% were sense overlapping IncRNAs, and 1.0\% were sense intronic IncRNAs (Fig. 2C). On the other hand, 1598 IncRNAs were filtered from the primary IncRNAs in SH-SY5Y cell and they contained $48.6 \%$ lincRNAs, $34.7 \%$ anti-sense IncRNAs, $16.0 \%$ sense overlapping IncRNAs, and $0.8 \%$ sense intronic IncRNAs (Fig. 2D).

Using Cuffdiff and $p<0.05$ as the criteria, the differentially-expressed IncRNAs in RD and SH-SY5Y cells in response to CV-B5 infection were shown in clustering analysis (Fig. 3A). Preliminary microarray hybridization in RD cell identified 508 up-regulated and 760 down-regulated IncRNAs which greatly changed after CVB5 infection (Fig. 3B). And, 792 significantly increased and 811 significantly decreased IncRNAs were identified in SH-SY5Y cell infected with CV-B5 (Fig. 3C). Among them, some have been associated with neurological disorders, such as TCONS_00385873, TCONS_00087939 and TCONS_00450969, when compared to RD cell. Therefore, we conclude that these IncRNAs may have critical roles in the nervous system.

\section{GO annotation and KEGG pathway analysis}

We used the clusterProfiler to explore the gene ontology functional classes and pathways in order to further understand our data. The differentially-expression IncRNAs were used to conduct the GO enrichment and KEGG pathway analysis. The top 20 significant $\mathrm{GO}$ terms of molecular function, biological process, and cellular component, based on the $p$ value and rich factors, were shown in Fig. 4. The results indicated that the most enriched $\mathrm{GO}$ terms in RD cell were single-organism process and membrane part, and cellular process (Fig. 4A). And cell part and protein binding were the most enriched GO terms in SH-SY5Y cell (Fig. 4B).

With regard to the KEGG pathway, the most enriched terms were mainly neuroactive ligand-receptor interaction, spliceosome, and viral myocarditis for CV-B5 infection in RD cell (Fig. 5A). On the other hand, the enriches terms in SH-SY5Y cell were mainly associated with several signaling pathways including ECM-receptor interaction, complement and coagulation cascades and TGF-beta signaling pathway (Fig. $5 B)$.

\section{RT-qPCR for validating the IncRNAs after CV-B5 infection}

To further verify the accuracy of the RNA sequencing analysis, the significantly up- and down- regulated IncRNAs were selected and validated using RT-qPCR. Seven IncRNAs were selected for further validation. 
Fold changes in the CV-B5 infected cells were calculated using the $2^{-\triangle \Delta C T}$ method (three independent experiments). Results showed that three IncRNAs were up-regulated and four IncRNAs were downregulated (Fig. 6). These findings were in accordance with our data analysis, which indicated that fold change results determined by RT-qPCR were lower than those determined using RNA sequencing results.

\section{The analysis of antisense IncRNA-IL12A}

The function of IncRNAs needs further experimentation because it cannot only be inferred from the RNA sequencing results. LncRNA-IL12A belonged to antisense IncRNA which related the gene Interleukin-12 subunit alpha and located on chromosome 3. In our present study, we selected IncRNA-IL12A in order to illustrate its roles in CV-B5 replication. Firstly, we predicted the secondary structure of IncRNA-IL12A (Fig. 7A). Then we contrasted pcDNA3.1-2Flag-IncRNA-IL12A transfect into 293T and found it does not have the ability to code (Fig. 7B). Next, we overexpressed and silenced its RNA expression in order to determine CV-B5 VP1 expression and our results showed that LncRNA-IL12A inhibits viral replication (Fig. 7CD). Finally, from the KEGG analysis we found the IncRNA-IL12A enriched in the Wnt signaling pathway and the key molecules downstream (C-myc and CyclinD1) siginicantly changes by real-time RT-PCR (Fig. 7E).

\section{Discussion}

In our research, we used the next-generation sequencing to investigate the non-coding transcriptome of CV-B5 infected RD and SH-SY5Ycells. The analyses revealed significant differences in the patterns of IncRNAs expression in different cells. In additional, we performed GO and KEGG analysis to determine the functions of these IncRNAs. Moreover, we explored the regulatory mechanism of the candidate IncRNAIL12A on virus replication and found that it inhibited viral replication through Wnt signaling pathway. To the best of our knowledge, this study is the first comprehensive research that has clearly illustrated the role of IncRNA in the host response to CV-B5 infection.

Since the development of a vaccine for EV-71, CV-B5 has been considered as the emerging neurotropic enteroviral pathogen and it has led to an increasing in the prevalence of severe HFMD [9-11]. Therefore it is vital to use a neuron-specific human cell in order to understand the interactions between CV-B5 and host. In this study, RD and SH-SY5Y cells were selected as experimental models. RD cell (derived from rhabdomyosarcoma) are highly susceptible to CV-B5 and often used to isolate CV-B5 from clinical specimens. Neuroblastoma cell line SH-SY5Y is an excellent model system for neurotropic viruses and is useful for neuropathogenicity studies and it has been previously used in the research of human neuronal cell for EV-71, Enterovirus D68, and Zika virus (Brown et al., 2018; Martín et al., 2018; Xu et al., 2013). Thus, we used the SH-SY5Y cell model to determine whether a barrier to viral entry was partly responsible for neurotropism. In this study, we successfully modeled the virus infection (Fig. 1), infect the RD and SHSY5Y cells with $1 \mathrm{MOI}$ CV-B5 at 24h, we observed the CPE and also measured the CV-B5 specific protein. 
Unlike mRNAs, IncRNAs have some unique and important characteristics. The RNA sequencing conducted identified 1268 differentially expressed IncRNAs in RD cell and 1603 differentially expressed IncRNAs in SH-SY5Y cell after CV-B5 infection. From the Heat map analysis, CV-B5 infected the different cells show that IncRNA expression profiles are significantly different (Fig. 3). We then used GO and KEGG pathway analysis to identify the differentially expressed genes. GO analysis that they were mainly enriched single-organism process and membrane part in RD cell and cellular process, cell part and protein binding in SH-SY5Y cell (Fig. 4). And KEGG results indicated that contractile fiber, myofibril, and chromatin assembly genes were enriched upon CVB5 infection in RD cell (Fig. 5A). On the other hand, embryonic morphogenesis, ossification, and membrane raft genes were enriched upon CV-B5 infection in SH-SY5Y cell, and the coding gene was enriched in the neurotrophin and ErbB signaling pathway (Fig. 5B). Previous studies have reported that the neurotrophin signaling pathway can regulate growth, differentiation, and apoptosis of neurons through p75 neurotrophin receptor and tyrosine kinase receptor [35; 36]. ErbB signaling pathway modulates neural development, cell proliferation, and differentitation and is also associated with neurodevelopment disorders [37]. The above-mentioned results suggest that the CV-B5 can lead to neurogenic diseases.

Similar to CV-B5, EV-71 and CV-A16 also belong to the human enterovirus genus within the Picornaviridae family and they are the main pathogens that cause HFMD and even severe neurological diseases [1]. Previous study had showed the IncRNA expression profile of CV-A16 and EV-71 infecting RD cell [38; 39]. Our results indicated that CV-B5 infected RD cell had the lowest number of total IncRNAs, 1268, while the EV-71 and CV-A16 infected RD cell had 3231 and 1970 IncRNAs, respectively. Among the total IncRNAs, intergenic IncRNAs accounted for about more than half of EV-71, CV-A16, and CV-B5 infection. Several reports have defined intergenic IncRNAs as a transcription brake that both promotes and represses the expression of inflammatory genes [40; 41]. Therefore, we speculate that EV-71, CV-A16, and CV-B5 having $50 \%$ of intergenic IncRNAs may regulate inflammation. However, antisense IncRNAs accounted for $28.6 \%$ of CV-B5 infection compared with $12.7 \%$ in EV-71 and 1.54\% in CV-A16 infection. Antisense IncRNAs can enhance combination of the promoter and enhancer responsible for regulating gene expression [42]. In our study, IncRNA-IL-12A belongs to antisense IncRNAs, but further verification should be conducted to determine whether it influences the promoter level. And KEGG analysis CV-A16 infection of RD cell showed that the significant difference was focused on PI3K-Akt and Wnt signaling pathway. On the other hand, KEGG analysis after CV-B5 infection of the cells revealed that the difference was concentrated on disease including viral myocarditis and leukocyte transendochelial migration.

Recently, a number of studies have showed that IncRNAs modulate pathogen-host interactions [43], Winterling et al. used NCode and Sureprint G3 systems in human lung epithelial cells and found that lincRNA VIN can facilitate the propagation of influenza A virus. However, the same results were not observed with influenza B virus [44]. Cao et al reported that IncRNA-ACOD1 can regulate recombinant GOT2 protein and its metabolites influence VSV replication [45]. In our study, we have also concluded that IncRNA-IL-12A can regulate CV-B5 infection by Wnt signaling pathway, but further verification on protein level should be conducted. Despite the progresses in the interaction between virus and IncRNAs, the 
specific functions of these IncRNAs have not been fully elucidated. Therefore, the virus infection models used in the study will provide a new platform for studying the mechanism and regulation of IncRNAs.

\section{Abbreviations}

CV-B5 Coxsackievirus B5

RD Human Rhabdomyosarcoma

GO Gene Ontology

KEGG Kyoto Encyclopedia of Genes and Genomes

RT-qPCR Real-time quantitative PCR

HFMD Hand, foot, and mouth disease

MOI Multiplicity of Infection

CPE Cytopathic Effect

hpi Hour Post-Infection

\section{Declarations}

\section{Author contributions}

WC designed the research and wrote the paper, PYT and HY performed the majority of experiments and analyzed the data, $\mathrm{JL}$ and FY participated in some experiments and prepared the figures and tables, WC and $\mathrm{HY}$ revised and edited the final paper.

\section{Funding}

This study was funded by the National Natural Science Foundation of China (No. 81860357), Young Talents Support Program of Yunnan Province (Ten Thousand People Plan, YNWR-QNBJ-2019-178), The State Key Laboratory of Virology (No. 2018KF006).

\section{Compliance with ethical standards}

\section{Conflict of interest}

None conflict of interest. 
Ethics approval The study does not involve animal and human samples.

\section{References}

1. Esposito S, Principi N (2018) Hand, foot and mouth disease: current knowledge on clinical manifestations, epidemiology, aetiology and prevention. Eur J Clin Microbiol Infect Dis 37:391-398

2. Jahan S, Liu SJ, Zhao H, Falak Z, ljaz U et al (2020) Neurological Complications Of Hand, Foot And Mouth Disease In Children: A Review. J Ayub Med Coll Abbottabad 32:562-569

3. Posnakoglou L, Tatsi EB, Chatzichristou P, Siahanidou T, Kanaka-Gantenbein C et al (2021) Molecular Epidemiology of Enterovirus in Children with Central Nervous System Infections. Viruses 13:100

4. Cabrerizo M, Tarragó D, Muñoz-Almagro C, Amo ED, Domínguez-Gil M et al (2014) Molecular epidemiology of enterovirus 71, coxsackievirus A16 and A6 associated with hand, foot and mouth disease in Spain. Clin Microbiol Infect 20:0150

5. Nhan LNT, Khanh TH, Hong NTT, Van HMT, Nhu LNT et al (2020) Clinical, etiological and epidemiological investigations of hand, foot and mouth disease in southern Vietnam during 20152018. PLoS Negl Trop Dis 14:e0008544

6. Gao F, Bian L, Hao XT, Hu YL, Yao X et al (2018) Seroepidemiology of coxsackievirus B5 in infants and children in Jiangsu province, China. Hum Vaccin Immunother 14:74-80

7. Tsuno K, Miyatake C, Nishijima H, Hotta C, Ogawa T et al (2018) Coxsackievirus B5 Aseptic Meningitis in Infants in Chiba Prefecture, Japan, in 2016. J Nippon Med Sch 85(3):187-190

8. Sciandra I, Falasca F, Maida P, Tranquilli G, Carlo DD et al (2020) Seroprevalence of group B Coxsackieviruses: Retrospective study in an Italian population. J Med Virol

9. Naveen T, Chris K (2021) Group B Coxsackieviruses. StatPearls

10. Liang XR, Shi YX, Zhang YH, Sun ZL, Zhang F et al (2018) Etiology and clinical analysis of central nervous system infection caused by Coxsackievirus B5 in severe hand, foot and mouth disease in Qingdao City, 2013-2014. Zhonghua Yu Fang Yi Xue Za Zhi 52:1168-1172

11. Hu YF, Yang F, Du J, Zhang T, Xue Y et al (2012) Coxsackievirus B5, associated with neurological hand, foot and mouth disease, China. J Infect 65:189-191

12. Jarroux J, Morillon A, Pinskaya M (2017) History, Discovery, and Classification of IncRNAs. Adv Exp Med Biol 1008:1-46

13. Liu KS, Mao XD, Chen YJ, Li TP, Ton H (2018) Regulatory role of long non-coding RNAs during reproductive disease. Am J Transl Res 10:1-12

14. Ferrè F, Colantoni A, Helmer-Citterich M (2017) Revealing protein-IncRNA interaction. Brief Bioinform 17:106-116

15. Paralkar VR, Weiss MJ (2013) Long noncoding RNAs in biology and hematopoiesis. Blood 121:4842-4846

16. Kopp F, Mendell JT (2018) Functional Classification and Experimental Dissection of Long Noncoding RNAs. Cell 172:393-407 
17. Basavappa M, Cherry S, Henao-Mejia JJ (2019) Long noncoding RNAs and the regulation of innate immunity and host-virus interactions. J Leukoc Biol 106:83-93

18. Robinson EK, Covarrubias S, Carpenter S (2020) The how and why of IncRNA function: An innate immune perspective. Biochim Biophys Acta Gene Regul Mech 1863:194419

19. Chai WJ, Li J, Shangguan QL, Liu Q, Li XD et al (2018) Lnc-ISG20 Inhibits Influenza A Virus Replication by Enhancing ISG20 Expression. J Virol 92:e00539

20. Chai WJ, Li J, Shangguan QL, Liu Q, Li XD et al (2019) Long noncoding RNA and its role in virus infection and pathogenesis. J Virol 92:e00539

21. Liu W, Wang ZQ, Liu L, Yang ZH, Liu S et al (2020) LncRNA Malat1 inhibition of TDP43 cleavage suppresses IRF3-initiated antiviral innate immunity. Proc Natl Acad Sci USA 117:23695-23706

22. Parkhomchuk D, Borodina T, Amstislavskiy V, Banaru M, Hallen L et al (2009) Transcriptome analysis by strand-specific sequencing of complementary DNA. J Nucleic acids research. Nucleic Acids Res 37:e123

23. Mortazavi A, Williams BA, McCue K, Schaeffer L, Wold B et al (2008) Mapping and quantifying mammalian transcriptomes by RNA-SEq. Nat Methods 5:621-628

24. Andrews S (2010) FastQC: A Quality Control tool for High Throughput Sequence Data. Reference Source

25. Garber M, Grabherr MG, Guttman M, Trapnell C (2011) Computational methods for transcriptome annotation and quantification using RNA-sEq. Nat Methods 8:469-477

26. Wright MM (2014) A short guide to long non-coding RNA gene nomenclature. Hum Genomics 8:7

27. Kang YJ, Yang DC, Kong L, Ho M, Meng YQ et al (2017) CPC2: a fast and accurate coding potential calculator based on sequence intrinsic features. Nucleic Acids Res 45:12-16

28. Luo H, Bu DC, Sun L, Chen RS, Zhao Y (2014) De novo approach to classify protein-coding and noncoding transcripts based on sequence composition. Methods Mol Biol 1182:203-207

29. Harrow J, Frankish A, Gonzalez JM, Tapanari E, Diekhans M et al (2012) GENCODE: the reference human genome annotation for The ENCODE Project. Genome Res 22:1760-1774

30. Trapnell C, Williams BA, Pertea G, Mortazavi A, Kwan G et al (2010) Transcript assembly and quantification by RNA-Seq reveals unannotated transcripts and isoform switching during cell differentiation.The Science and Business of Biotechnology. Nat Biotechnol 28:511-515

31. McDermaid A, Monier B, Zhao J, Liu B, Ma Q (2019) Interpretation of differential gene expression results of RNA-seq data: review and integration. Brief Bioinform 20(6):2044-2054

32. Harrow J, Frankish A, Gonzalez JM, Tapanari E, Diekhans M et al (2012) GENCODE: the reference human genome annotation for The ENCODE Project. Genome Res 22:1760-1774

33. Trapnell C, Williams BA, Pertea G, Mortazavi A, Kwan G et al (2010) Transcript assembly and quantification by RNA-Seq reveals unannotated transcripts and isoform switching during cell differentiation.The Science and Business of Biotechnology. Nat Biotechnol 28:511-515 
34. Blake JA, Harris MA (2008) The Gene Ontology (GO) Project: Structured Vocabularies for Molecular Biology and Their Application to Genome and Expression Analysis. Curr Protoc Bioinformatics 7

35. Du JL, Yuan ZF, Ma ZW, Song JZ, Xie XL et al (2014) KEGG-PATH: Kyoto encyclopedia of genes and genomes-based pathway analysis using a path analysis model. Mol Biosyst

36. Gong Y, Cao P, Yu HJ,Jiang T (2008) Crystal structure of the neurotrophin-3 and p75NTR symmetrical complex. Sci Rep 454:789-793

37. Kizilyer A, Singh MV, Singh VB, Suwunnakorn S, Palis J et al (2019) Inhibition of Tropomyosin Receptor Kinase A Signaling Negatively Regulates Megakaryopoiesis and induces Thrombopoiesis. Sci Rep 9:2781

38. Britsch S (2007) The neuregulin-I/ErbB signaling system in development and disease. Adv Anat Embryol Cell Biol 190:1-6541

39. Li Y, Zhang C, Qin L, Li D, Zhou G et al (2018) Characterization of Critical Functions of Long NonCoding RNAs and mRNAs in Rhabdomyosarcoma Cells and Mouse Skeletal Muscle Infected by Enterovirus 71 Using RNA-Seq. Viruses

40. Castellanos-Rubio A, Fernandez-Jimenez N, Kratchmarov R, Luo XB, Bhagat G et al (2016) A long noncoding RNA associated with susceptibility to celiac disease. Science 352:91-95

41. Atianand MK, Hu W, Satpathy AT, Shen Y, Ricci EP et al (2016) A Long Noncoding RNA lincRNA-EPS Acts as a Transcriptional Brake to Restrain Inflammation. Cell 165:1672-1685

42. Canzio D, Nwakeze CL, Horta A, Rajkumar SM, Coffey EL et al (2019) Antisense IncRNA Transcription Mediates DNA Demethylation to Drive Stochastic Protocadherin a Promoter Choice. Cell 177:639653

43. Liu WW, Ding C (2017) Roles of LncRNAs in Viral Infections. Front Cell Infect 7:205

44. Winterling C, Koch M, Koeppel M, Garcia-Alcalde F, Karlas A et al (2014) Evidence for a crucial role of a host non-coding RNA in influenza A virus replication. RNA Biol 11:66-75

45. Wang P,XuJ, Wang Y,Cao X (2017) An interferon-independent IncRNA promotes viral replication by modulating cellular metabolism. Science 358:1051-1055

\section{Figures}


A

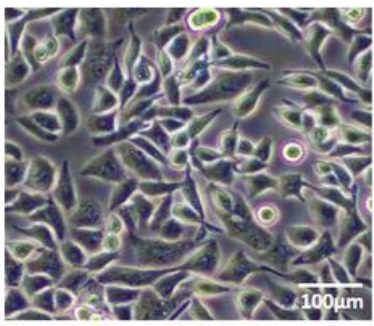

RD-Con

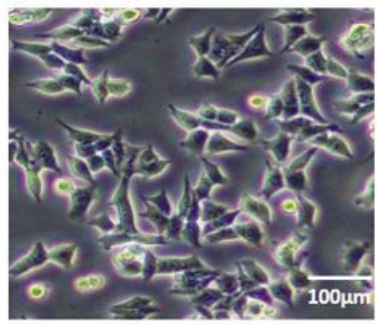

SH-SY5Y-Con

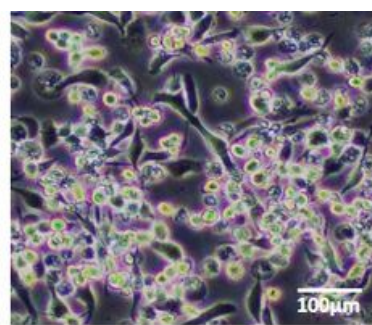

RD-CVB5

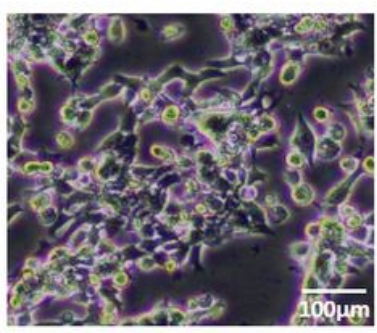

SH-SY5Y-CVB5
B
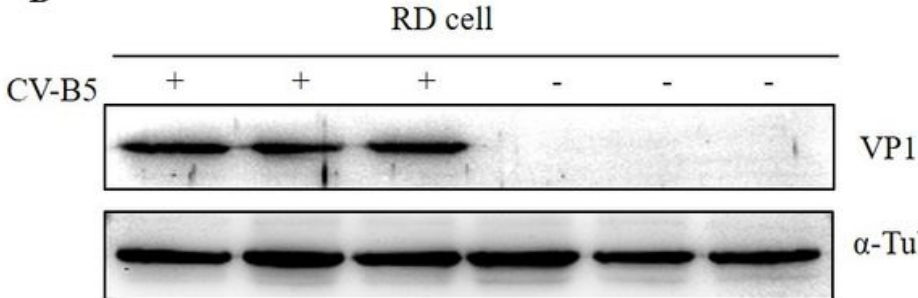
$\alpha$-Tubulin

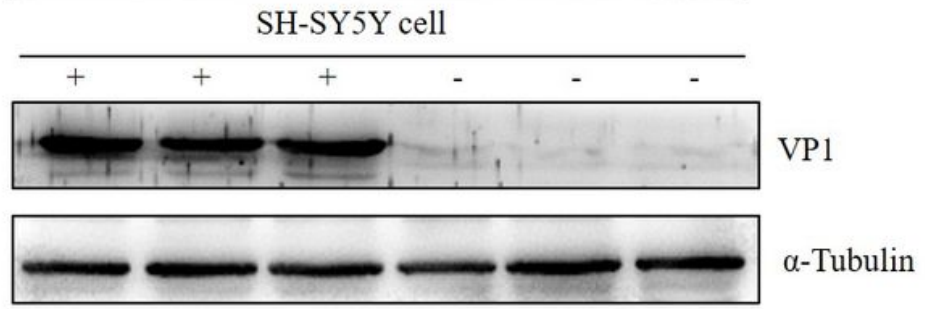

\section{Figure 1}

RD and SH-SY5Y cell models infected with 1MOI CV-B5 for 24h. (A) CPE in RD and SH-SY5Y cells were captured using light microscopy (amplification: $\times$ ) compared to control cells; (B) Expression of CV-B5 specific protein (VP1) was validated in RD and SH-SY5Y cells using Western Blot analysis.

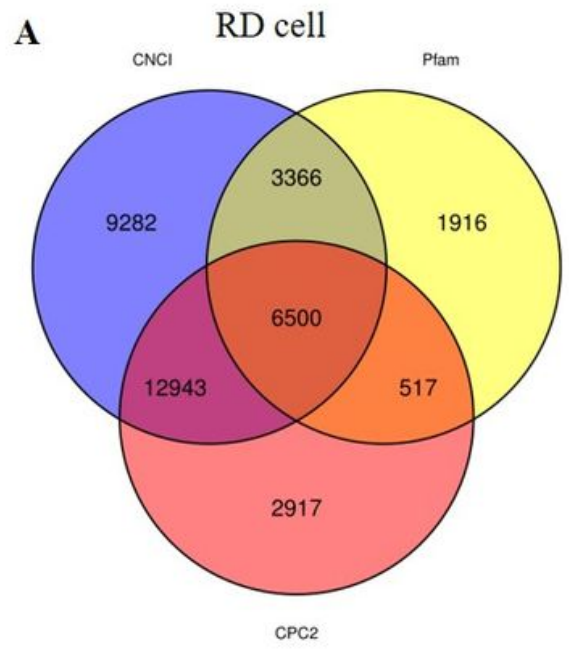

B

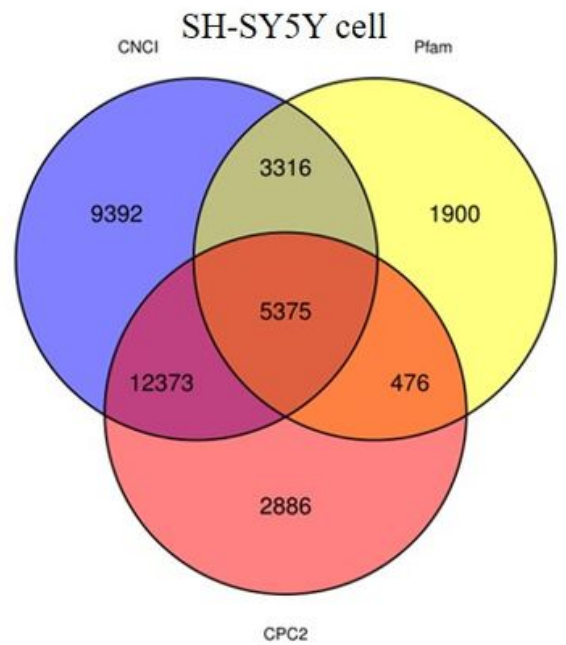

C RD cell

D

SH-SY5Y cell

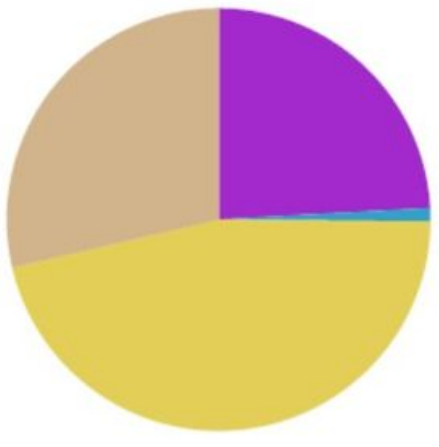

sense_intronic(1.0\%)

antisense $(28.6 \%)$

sense_overlapping(24.1\%)

lincRNA(46.2\%)

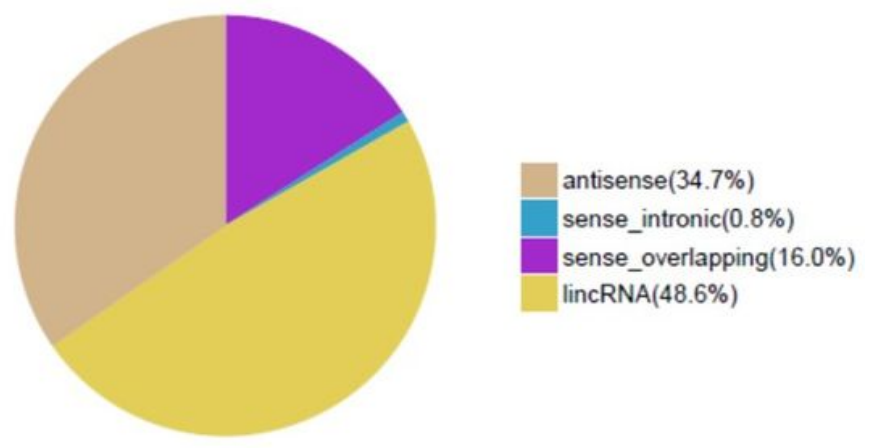

Figure 2 
Classification and characteristics of IncRNAs. (A/B) Novel IncRNAs in RD and SH-SY5Y cells according to the $\mathrm{CPC} 2, \mathrm{CNCl}$, and PFAM analysis; (C/D) Venn diagram showing the classification of differentially expressed genes in RD and SH-SY5Y (5Y) cells.
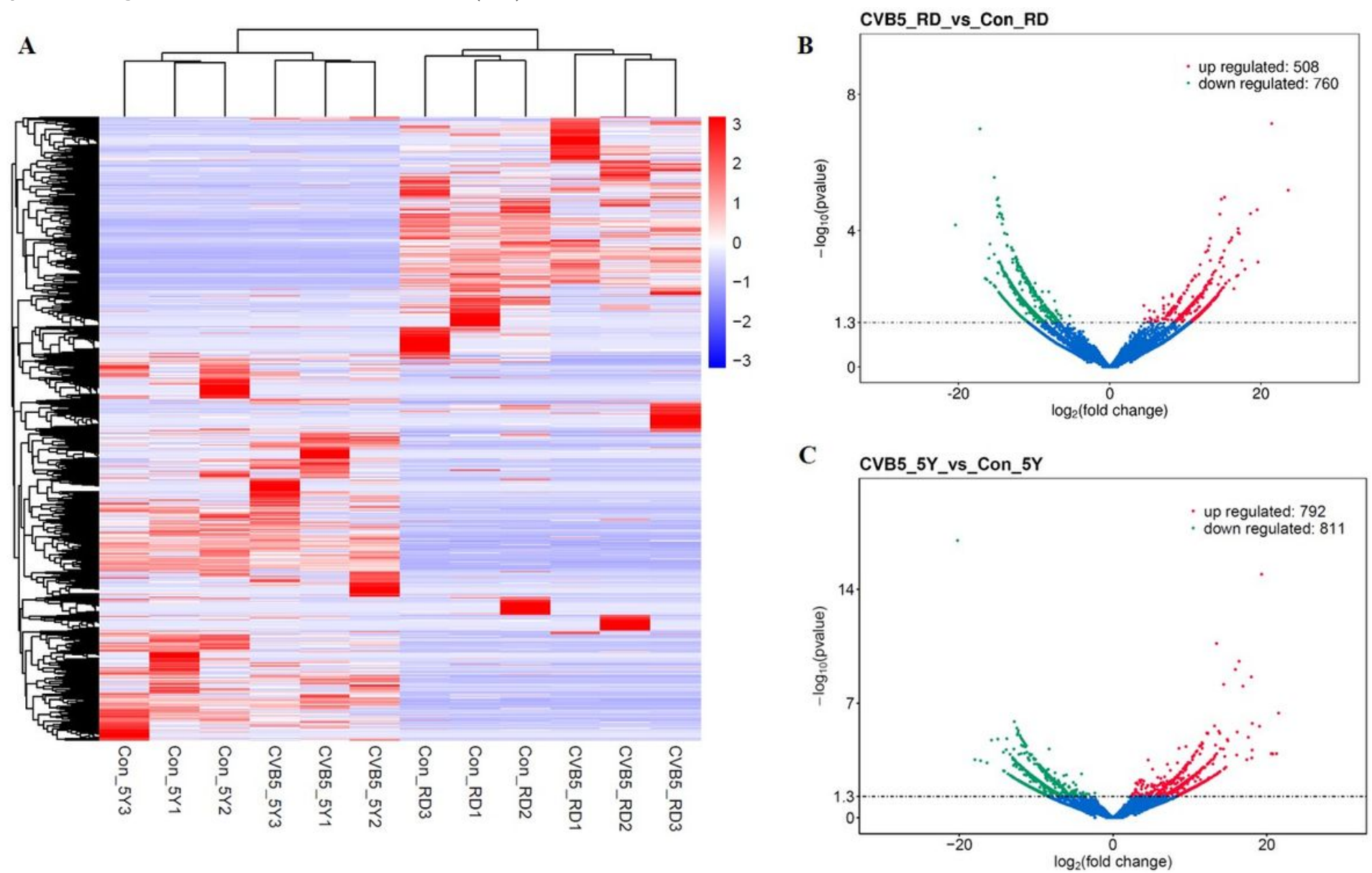

\section{Figure 3}

Heatmap showing differential expression of IncRNAs in RD and SH-SY5Y cells infected with CV-B5. (A) Cluster analysis of differentially expression of IncRNA in all samples; (B) Analysis of differentially expression of IncRNA in RD cell after CV-B5 infection; (C) Analysis of differentially expression IncRNA in SH-SY5Y (5Y) cell after CV-B5 infection; 
A

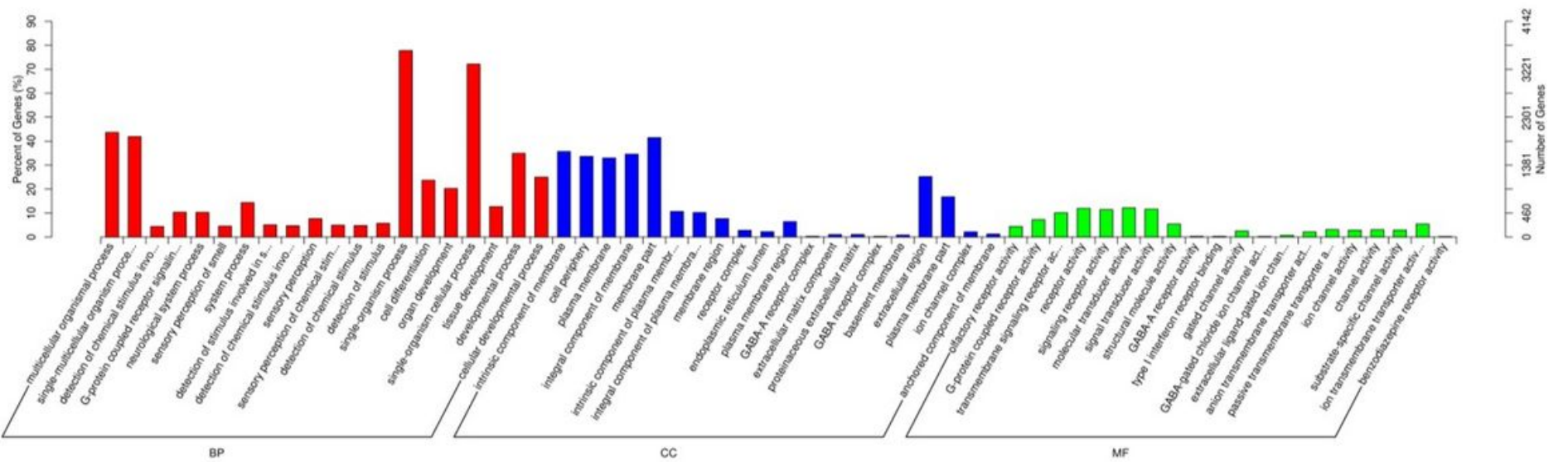

\section{B}

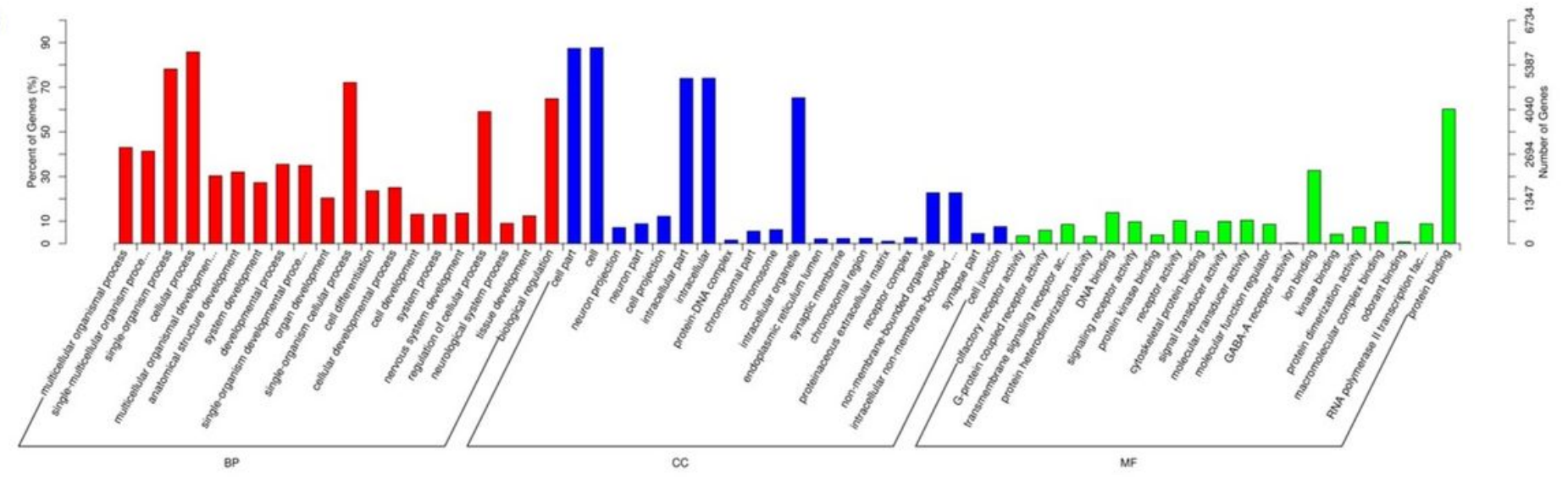

Figure 4

GO analysis of differentially-expressed genes after CV-B5 infection. (A/B) The top 20 significantly enriched GO terms in differentially-expressed of IncRNAs in CV-B5 infected RD and SH-SY5Y (5Y) cells.
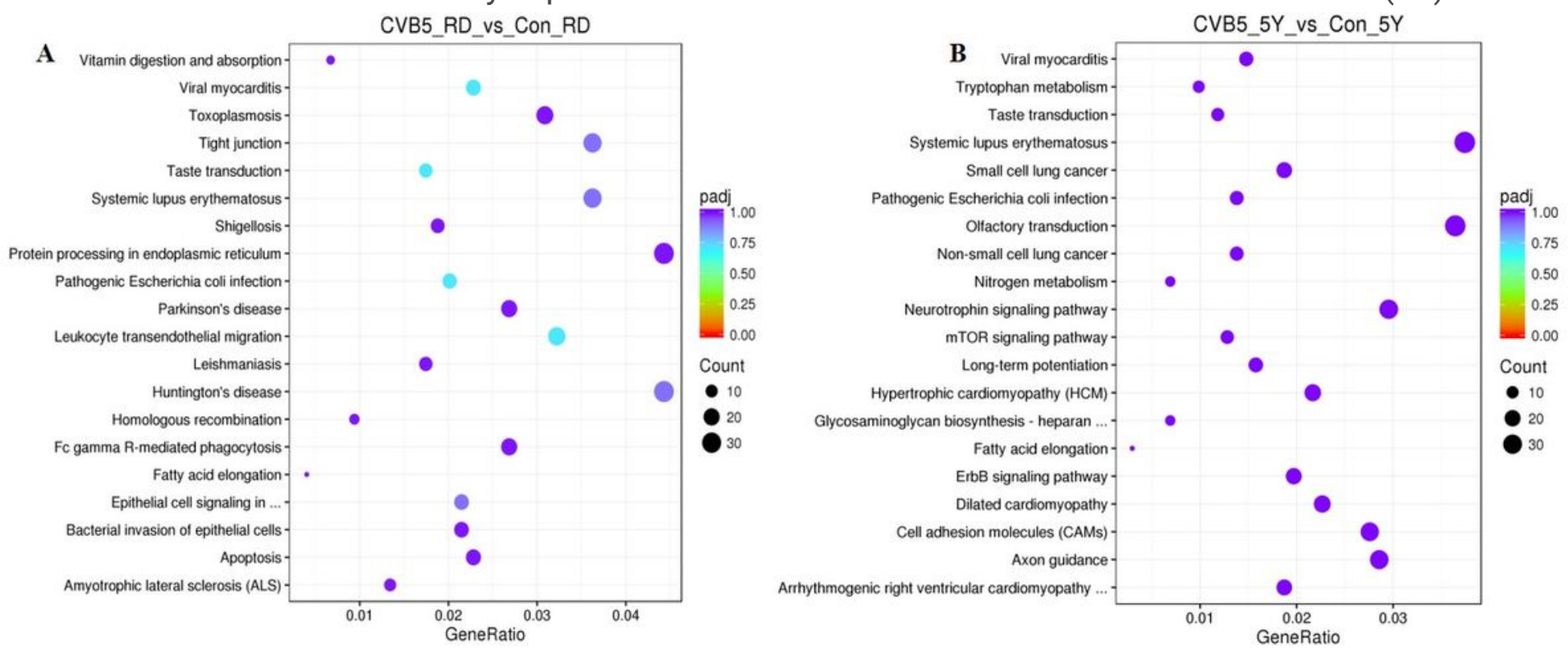

Figure 5 
The KEGG pathway analysis of differentially-expressed genes after CV-B5 infection. (A/B) The top 20 enriched pathways associated with differentially-expressed IncRNAs in RD and SH-SY5Y (5Y) cells.

A

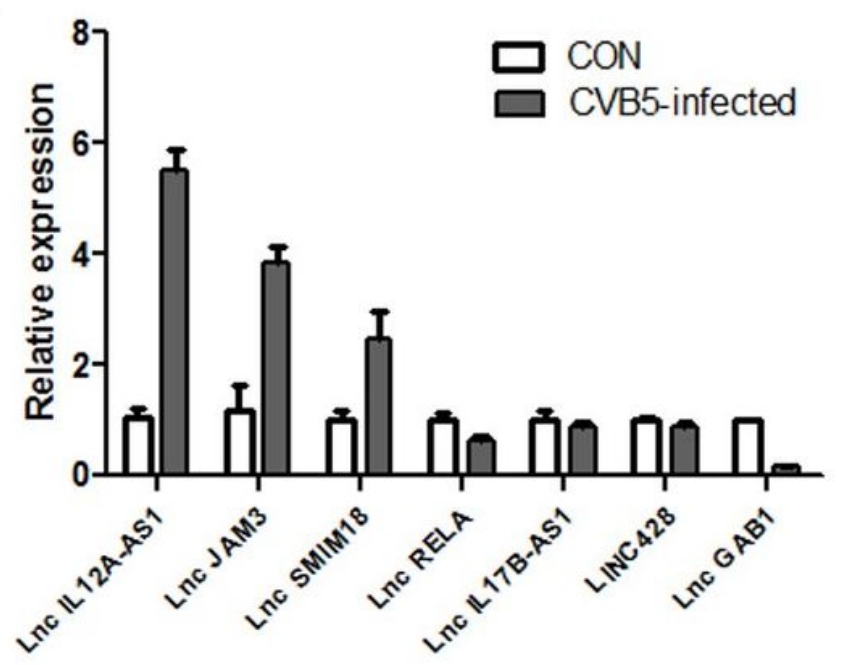

B

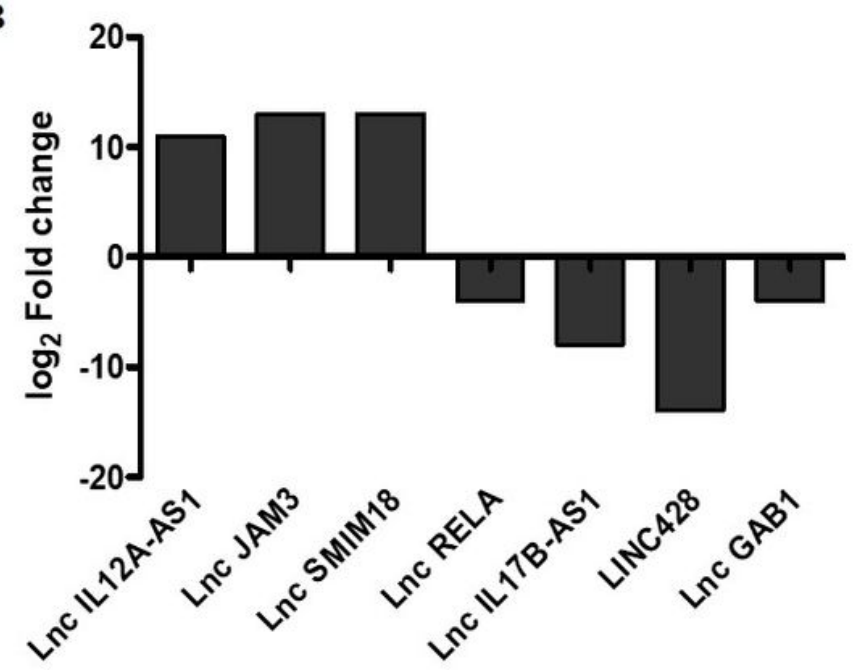

Figure 6

The expression of seven differentially expressed IncRNAs was validated using qRT-PCR and RNA-seq results. (A) The validation results of RT-qPCR; (B) The primary results of RNA-seq. 
A

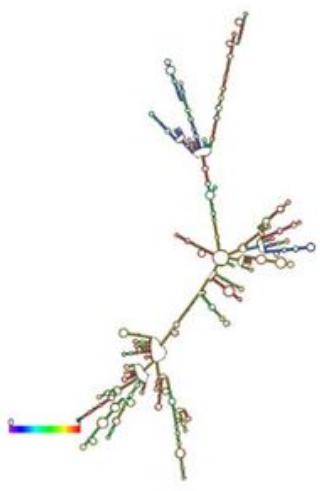

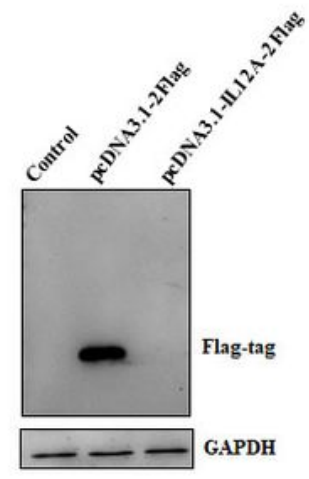

C

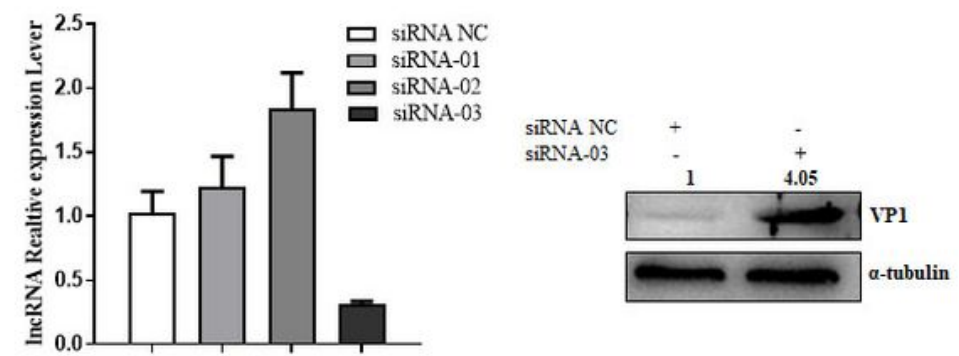

D

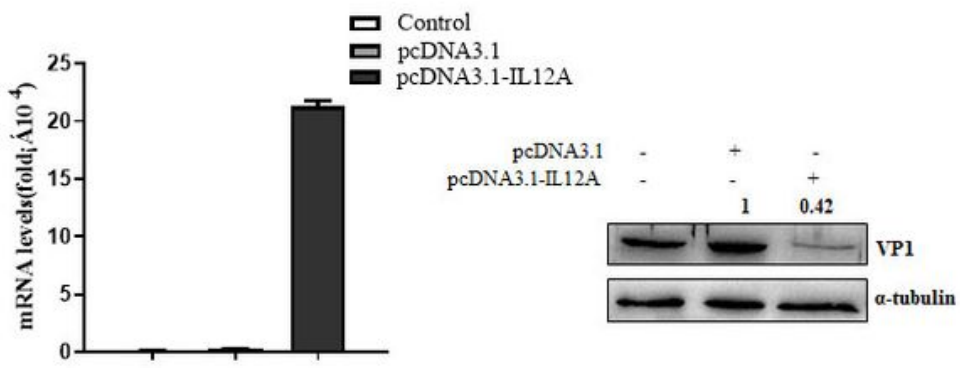

E

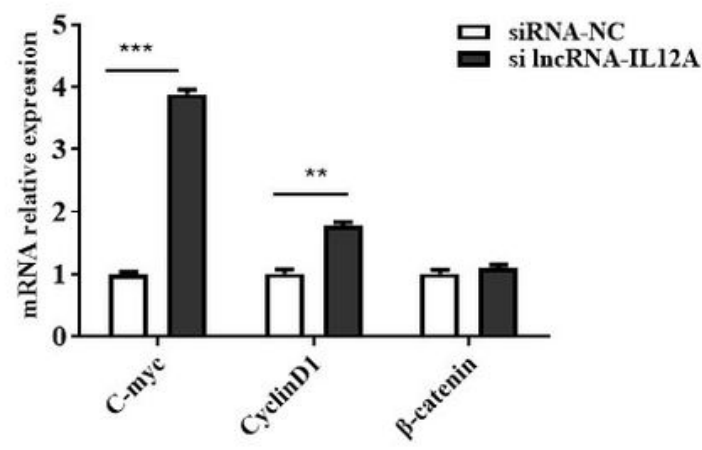

Figure 7

The inhibition of virus replication by IncRNA-IL12A. (A) The secondary structure of IncRNA-IL12A; (B) Coding ability of IncRNA-IL12A verification; (C) qPCR analysis the expression of IncRNA-IL12 with SHSY5Y cell was transfected with siRNA IncRNA IL12A (left); Western blot analysis of CV-B5 VP1 levels in SH-SY5Y cell transfected with siRNA IncRNA-IL12A, followed by infection CVB5 (right); (D) qPCR analysis the expression of IncRNA-IL12 with SH-SY5Y cell was transfected with control or pcDNA3.1-IncRNA IL12A 
(left); Western blot analysis of CV-B5 VP1 levels in SH-SY5Y cell transfected with pcDNA3.1-IncRNAIL12A, followed by infection CV-B5 (right); (E) IncRNA-IL12A inhibits the expression of downstream target genes (C-myc and CyclinD1) in the Wnt signaling pathway.

\section{Supplementary Files}

This is a list of supplementary files associated with this preprint. Click to download.

- Supplementarytable1.xlsx

- Supplementarytable2.xlsx 